\title{
Design Considerations For Low Field Short Photo-Injected RF Electron Gun With High Charge Electron Bunch
}

\author{
Xiangyun Chang, Ilan Ben-Zvi, Jörg Kewisch \\ Collider-Accelerator Department, Brookhaven National Laboratory \\ Upton NY 11973 USA
}

\begin{abstract}
The RF field and space charge effect in a low field RF gun is given. The cell lengths are modified to have maximum accelerating efficiency. The modification introduces an extra RF field slice emittance. The phase space evolution of the following emittance compensation system is presented taking into account the chromatic effect. The emittance compensation mechanics for RF field and chromatic effect induced emittance is similar to that of compensating the space charge induced emittance. But the requirements are different to have best compensation for them. The beam waist is far in front of linac entrance to have best compensation for the RF field and chromatic effect induced emittance. For low field RF gun with high charge electron bunch this compensation is more important.
\end{abstract}

\section{INTRODUCTION}

The photo-injected RF gun system is normally composed by a RF gun and followed by a emittance compensation system. After that the electron energy is boosted by linacs to required energy.

Due to the time dependent $R F$ field, position related space charge force and position related beam energy the transverse motions of different slices in a beam bunch are different. This relative transverse slice motion forms a much bigger emittance of the beam as a whole compared to that of a single slice. The emittance compensation mechanics [1] [2] [3] is used to correct the relative transverse slice motion before the emittance is frozen by acceleration in linacs.

The RF and space charge effect in the RF gun is analytically given by Kwang-Je Kim [4]. In his theory the cell lengths inside the gun is assumed to be a half cell bounded at one side by the cathode and followed by a number of full cells (see fig.1(A)). This is suitable when the RF field in gun is relatively strong. But in some applications the system needs working at CW state, so limited by RF power and cavity heating problems the RF field can not be very high and the cell number is small. In this case, the above theory fails. The cell lengths should be accordingly modified to acquire highest accelerating efficiency and lowest emittance at gun exit (see fig.1(B)). The modification introduces some new beam properties. 
It is necessary to apply the emittance compensation mechanics to get low emittance. As the energy spread of the beam in the low field RF gun is non-negligible, the chromatic effect must take into account in the emittance compensation process.
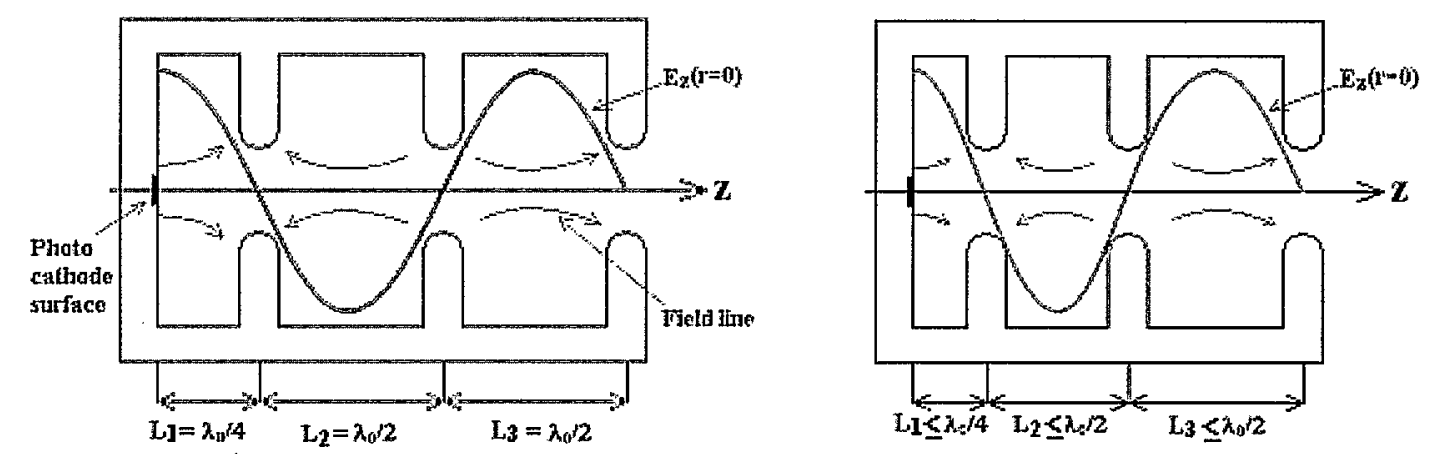

FIGURE 1. Schematics of the RF laser gun. (A): Standard $1 / 2$ cell $+\mathbf{n}$ cell gun for high field gun. (B): $1 / 2$ cell $+n$ cell gun for low field gun. The cell length for each cell is less than corresponding cell in (A).

\section{LONGITUDINAL MOTION}

We follow the line of thought in reference [4]. The electric field along the axis will be assumed to be of the following form for the low electric field case of fig.1(B):

$$
E_{z}=E(z) \cos \left(\int_{0}^{z} k(z) d z\right) \sin \left(\omega t+\phi_{0}\right) \text {. }
$$

Here $\mathrm{E}_{0}$ is the peak accelerating field, $\omega=c k_{0}, k_{0}=2 \pi / \lambda_{0}, \lambda_{0}$ is the RF wavelength and $\mathrm{c}$ is the speed of light, $\mathrm{k}(\mathrm{z})$ is defined as $k(z)=8 \pi / L$ for the $1^{\text {st }}$ half cell and $k(z)=4 \pi / L$ for the rest cells. $\mathrm{L}$ is cell length and should be modified according to average $\beta$ of electron in the cell to have maximum acceleration and minimum emittance (explain later). $\phi_{0}$ is the initial phase of electron on cathode. $\mathrm{E}(\mathrm{z})=\mathrm{E}_{0}=$ const. inside the gun and is $\mathrm{E}(\mathrm{z})=0$ outside the gun.

It is convenient to introduce the following quantity:

$$
\phi=\omega t-\int_{0}^{z} k(z) d z+\phi_{0}=\int_{0}^{z}\left(\frac{\gamma k_{0}}{\sqrt{\gamma^{2}-1}}-k(z)\right) d z+\phi_{0} .
$$

We have from (1) \& (2):

$$
\frac{d \gamma}{d z}=\frac{e E_{0}}{2 m c^{2}}\left[\sin \phi+\sin \left(\phi+2 \int_{0}^{z} k(z) d z\right)\right]
$$

The acceleration of electron in gun is determined by the pair of equations (2) and (3). From equation (3) it's clear that the maximum energy gain is obtained when $\phi$ is $\pi / 2$. $\phi_{0}$ should not be too small for the consideration of Schottky effect and strong space charge effect near cathode. For low electric field case, assume $\mathrm{k}(\mathrm{z})=\mathrm{k} 0=$ const. as for the high field case in reference [4]. From equation (2) we see that $\phi$ starts from $\phi_{0}$ on cathode and increases quickly near cathode where $\beta \& \gamma$ is still small. At end of the $1^{\text {st }}$ half cell, the integration in (2) can be big. After that, $\gamma$ is bigger and bigger and the 
integration doesn't increase much. As a result, $\phi$ approaches its asymptotic value which can be more than $\pi / 2$. Therefore the final energy is not maximum. Fig.2 shows the dependence of $\phi$ on normalized field strength $\alpha\left(\alpha=\frac{e E_{0}}{2 m c^{2} k_{0}}\right)$ and initial phase $\phi_{0}$. For low field case $(\alpha=0.5), \phi$ at gun exit $\phi_{\mathrm{f}}$ is always larger than $\pi / 2$.

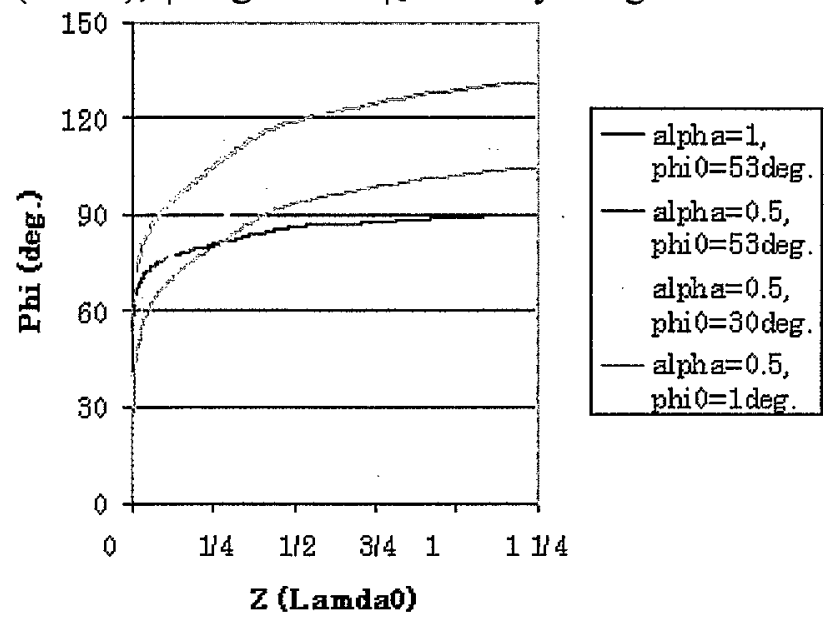

FIGURE 2. $\phi$ as a function of $\mathrm{z}$ for a $2 \&$ half cell cavity. Blue line: $\alpha=1$ (high field). $\phi_{0}=53 \mathrm{deg}$ to have $\phi_{\mathrm{f}}=90 \mathrm{deg}$. Magenta line: $\alpha=0.5$ (low field). $\phi_{\mathrm{f}}=131 \mathrm{deg}$ with $\phi_{0}=53 \mathrm{deg}$. Yellow line: $\alpha=0.5 . \phi_{\mathrm{f}}=111 \mathrm{deg}$ with $\phi_{0}=30 \mathrm{deg}$. Cyan line: $\alpha=0.5$. $\phi_{\mathrm{f}}=111 \mathrm{deg}$ with $\phi_{0}=1 \mathrm{deg}$.

To avoid $\phi$ being bigger than $\pi / 2$ we need increase $\mathrm{k}(\mathrm{z})$ according $\beta \& \gamma$ in cell. The lower the $\beta \& \gamma$, the shorter the cell. The simulation tells us that when $\phi$ at gun exit $\phi_{\mathrm{f}}=\pi / 2$ the electron energy is maximum and energy spread is minimum. A numerical simulation can be used to determine $\mathrm{k}(\mathrm{z})$. For example, when $\alpha=0.5, \phi_{0}=30 \mathrm{deg}$, by modifying the cell lengths, we make $\phi \approx \pi / 2$ after the $1^{\text {st }}$ half cell

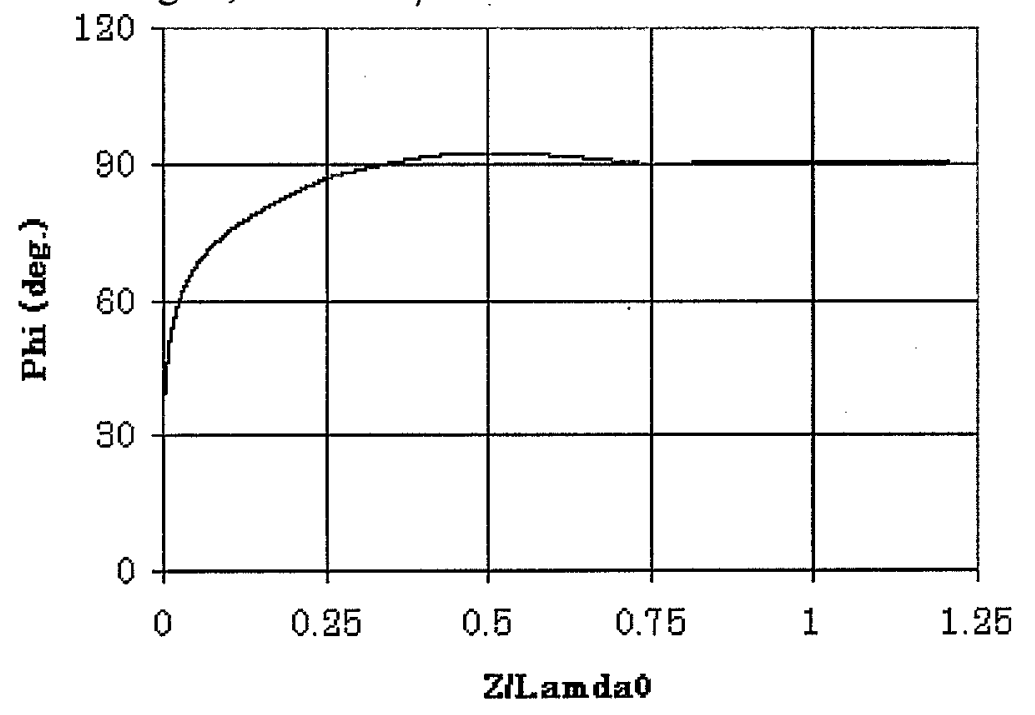

FIGURE 3. The cell lengths for $\alpha=0.5 . L_{1}=\lambda_{0} / 4 . L_{2}=\lambda_{0} /(2 \times 1.09) . L_{3}=\lambda_{0} /(2 \times 1.034)$.

It is found by simulation that after the cell length modification shown above not only the beam acquires maximum energy but also the minimum energy spread. 


\section{TRANSVERSE MOTION}

Let the longitudinal electric field $E_{z}$ be a function of only $z$ and $t$ and be independent of the transverse coordinates $r$ and angle $\theta$. From Maxwell's equations we have:

$$
E_{r}=-\frac{r}{2} \frac{\partial E_{z}}{\partial z}, \quad c B_{\theta}=\frac{r}{2 c} \frac{\partial E_{z}}{\partial t}
$$

The radial force acting on an electron is given by:

$$
F_{r}=e\left(E_{r}-\beta c B_{\theta}\right)
$$

Inserting (1) into above equation we get:

$$
\begin{aligned}
& F_{r}=e r\left\{-\frac{1}{2 c} \frac{d}{d t}\left[E(z) \sin \left(\int_{0} k(z) d z\right) \cos \left(\omega t+\phi_{0}\right)\right]\right. \\
& +\frac{\beta}{2} \frac{d E(z)}{d z} \sin \left(\int k(z) d z\right) \cos \left(\omega t+\phi_{0}\right)-\frac{1}{2} \frac{d E(z)}{d z} \cos \left(\int_{0} k(z) d z\right) \sin \left(\omega t+\phi_{0}\right) \\
& +\frac{\beta}{2}\left(k(z)-k_{0}\right) E(z) \cos \left(\int_{0}^{z} k(z) d z\right) \cos \left(\omega t+\phi_{0}\right) \\
& \left.+\frac{1}{2}\left(k(z)-k_{0}\right) E(z) \sin \left(\int_{0}^{\pi} k(z) d z\right) \sin \left(\omega t+\phi_{0}\right)\right\}
\end{aligned}
$$

The radial motion is described by:

$$
\frac{d p_{r}}{d t}=\frac{1}{m c} F_{r}, \quad p_{r}=\gamma \frac{1}{c} \frac{d r}{d t}
$$

Where $P_{r}$ is the dimensionless radial momentum. We integrate (7) to get $P_{r}$. Assume $r$ is constant, the $1^{\text {st }}$ term in (6) is a total time derivative of an expression that vanishes on cathode surface and at the outside of the cavity, its integral is 0 . The contribution of $2^{\text {nd }}$ term and $3^{\text {rd }}$ term comes only from the region where $\mathrm{dE}(\mathrm{z}) / \mathrm{dz}$ is nonzero, i.e., near the cavity exit. We take $\mathrm{E}(\mathrm{z})$ to be the form:

$$
E(z)=\theta\left(z_{f}-z\right) E_{0}
$$

Where $\theta$ is the step function and $\mathrm{z}_{\mathrm{f}}$ is the position of cavity exit. Insert (8) into (6) the contribution of $2^{\text {nd }}$ and $3^{\text {rd }}$ terms $\mathrm{p}_{\mathrm{r}}(2,3)$ is:

$$
p_{r}(2,3)=p_{r 0}+\alpha k_{0} r\left[\beta \cos \left(\int_{0}^{s} k(z) d z\right) \sin \left(\omega t+\phi_{0}\right)-\sin \left(\int_{0}^{s} k(z) d z\right) \cos \left(\omega t+\phi_{0}\right)\right]
$$

Assume $\mathrm{p}_{\mathrm{r}}=0$ at $\mathrm{t}=0$ and since $\beta \approx 1$ at cavity exit, equation (9) becomes:

$$
p_{r}(2,3)=\alpha k_{0} r \sin \phi_{f}
$$

Where $\phi_{\mathrm{f}}$ is the $\phi$ value at gun exit. The above analysis is similar to those in [4] but with the replacement of $\mathrm{k}_{0} \mathrm{z}$ in equation (2) of reference [4] with $\int_{0}^{f} k(z) d z$. The beam has maximum divergence when $\phi_{\mathrm{f}}=\pi / 2$ but minimum slice emittance.

We also have extra $4^{\text {th }}$ and $5^{\text {th }}$ terms in (6). To show the contribution of these 2 terms, let's look at the example in fig.3. As the wave number in the $1^{\text {st }}$ half cell $\mathrm{k}_{1}=\mathrm{k}_{0}$, the integration starts from the $2^{\text {nd }}$ cell where $\beta \sim 1$. So these 2 terms can be written as: 


$$
F_{r}(4,5) \approx \frac{e r}{2}\left(k(z)-k_{0}\right) \cos \phi
$$

The divergence contribution of (11) is:

$$
p_{r}(4,5) \approx \alpha k_{0} r\left[\left(k_{2}-k_{0}\right) L_{2}+\left(k_{3}-k_{0}\right) L_{3}\right] \cos \phi=\alpha k_{0} r A(k) \cos \phi
$$

Where $\mathrm{A}(\mathrm{k})$ is a function of wave number $\mathrm{k}$. For the example in fig. $3, \mathrm{~A}(\mathrm{k}) \sim 0.78$. The beam divergence contribution from this term is small because $\phi \approx \pi / 2$ but the slice emittance contribution is maximum. For the example in fig. 3 we have:

$$
\frac{\partial p_{r}(4,5)}{\partial \phi}=-0.78 \alpha k_{0} r \sin \phi
$$

In general, the smaller the initial phase $\phi_{0}$ is, the smaller $\phi$ will be. To the $1^{\text {st }}$ order approximation, we assume that the divergence is a linear function of $\phi_{0}$ where the smaller the $\phi_{0}$ is (head of beam bunch), the larger the divergence.

For a general longitudinal Gaussian distribution of a bunch the space charge force is maximum in the middle of the bunch and minimum at its ends. The divergence as a function of position in beam bunch at cavity exit is schematically shown in fig.4.

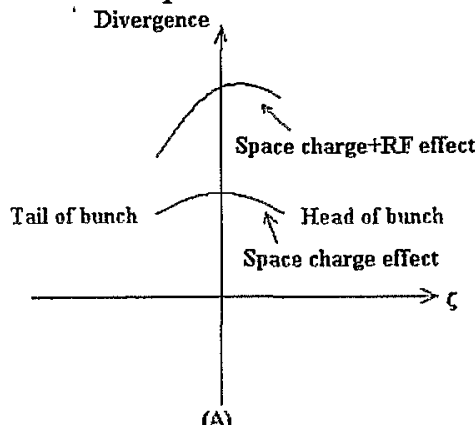

(A)

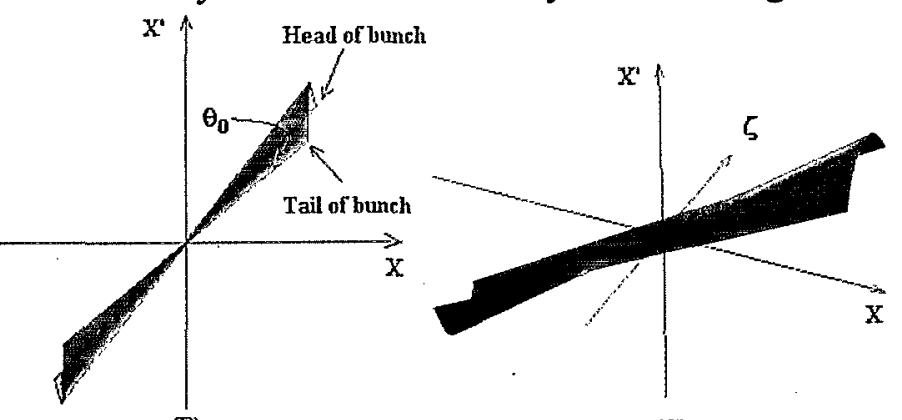

(C)

FIGURE 4. Divergence as a function of relative position $\zeta$ of a bunch $(\zeta=0$ represents center of bunch). (A): In $\zeta$-divergence space. (B): Corresponding phase space. $\theta_{0}$ is caused by the cell modification effect. $\theta_{0} \approx A(k) \alpha k_{0} \sigma_{\phi}$. (C): 3-D phase space.

\section{EMITTANCE COMPENSATION}

The emittance-compensation mechanism for a non-magnetized beam is discussed in many papers. Especially, B. E. Carlsten in reference [1], Luca Serafini in reference [2] and S. G. Anderson \& J. B. Rosenzweig in reference [3] gave excellent descriptions of emittance compensation mechanics. But these papers don't touch the chromaticity effect. Simply saying it is that by using the space charge force to align the different divergences between slices before the bunch is 'frozen' by acceleration. The system is simply composed by a short solenoid followed by a drift space and a linac (fig.5).

For our low field case, as the energy spread is high the chromatic effect must be taken into account. Consider a zero emittance beam with $x_{0}^{\prime}$ and non zero $\mathrm{x}_{0}$ passing through the thin lens. We have $\mathrm{x}=\mathrm{x} 0$ and $x^{\prime}=x_{0}^{\prime}+\Delta x^{\prime}$ after the lens:

$$
\Delta x^{\prime}=\frac{x_{0}}{f\left(1+\Delta p / p_{0}\right)} \approx \frac{x_{0}}{f_{\text {Sol }}}\left(1-\Delta p / p_{0}\right)
$$


Where $\Delta p / p_{0} \approx \Delta E / E_{0}$ is the momentum spread and $\mathrm{f}_{\mathrm{Sol}}$ is the focal length of the short solenoid.
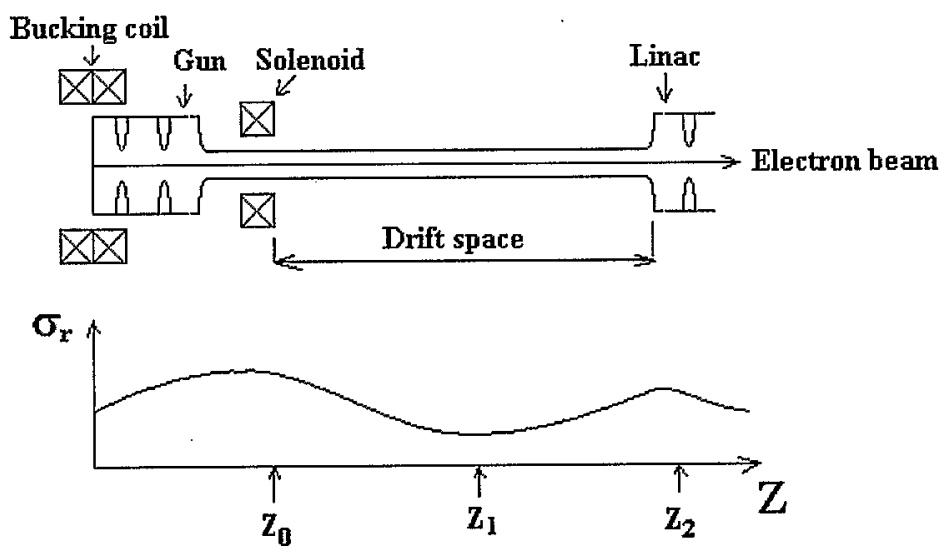

FIGURE 5. Emittance compensation system. $z_{0}$ is the position of the solenoid end, $z_{1}$ is the beam waist and $z_{2}$ is the mirror point of $z_{0}$ to $z_{1} \cdot z_{2}-z_{1}=z_{1}-z_{0}$.

To have the best emittance at linac exit we must also include the effect of linac. As we know that there is a strong focusing at entrance of linac. The effect can be treated as a short lens with focus length $f_{\text {Linac }}$ :

$$
\frac{1}{f_{\text {Linac }}} \approx \int_{z_{A}}^{z_{B}} \frac{\gamma^{\prime \prime}}{2 \beta^{2} \gamma} d z \approx \frac{\gamma^{\prime}}{2 \beta^{2} \gamma}
$$

Where $\mathrm{z}_{\mathrm{A}}$ is before linac entrance and $\mathrm{z}_{\mathrm{B}}$ is after linac entrance. The effect of linac entrance in the phase space is shown in fig.6.

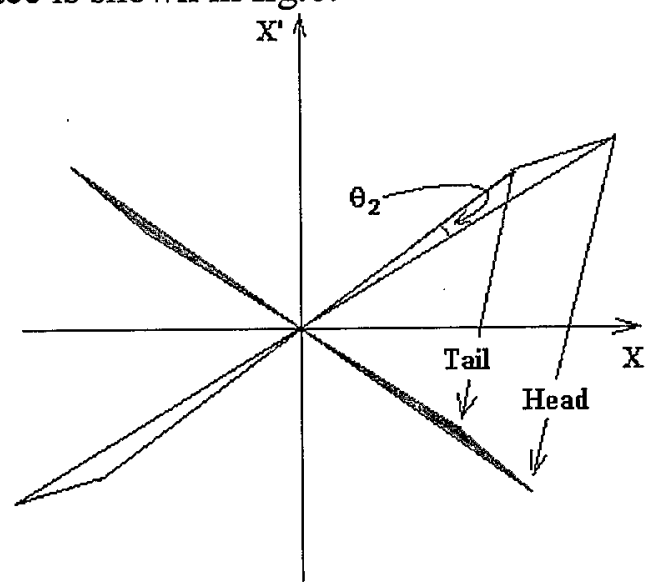

FIGURE 6. Focusing effect of linac entrance. $\theta_{2}$ is the divergence difference between high energy slice and low energy slice at the linac entrance.

The linac entrance induced angle change between low enrgy slice and high energy slice is:

$$
\Delta \theta_{\text {Linac }} \approx \frac{\gamma^{\prime}}{2 \beta^{2} \gamma} \frac{\Delta p}{p}
$$

To have minimum RF field and chromatic effect induced emittance this angle should be equal to $\theta_{2}$ in fig.6. Assume all slices form the waists at same position we have: 


$$
\left.\Delta \theta_{\text {Linac }}=\frac{\gamma^{\prime}}{2 \beta^{2} \gamma} \frac{\Delta p}{p}=\theta_{2} \approx \theta_{1}\left(\frac{z-z_{1}}{z_{1}-z_{0}}\right)^{2} \approx\left(A \alpha k_{0} \sigma_{z}+\frac{\Delta p / p}{f_{\text {Sol }}}\right)\left(\frac{z-z_{1}}{z_{1}-z_{0}}\right) 17\right)
$$

Where $z$ is the location of linac entrance and is larger than $z_{1}$ in fig.5. For higher linac field gradient, equation (17) requires higher A (i.e., more cell length reduction) or stronger solenoid or bigger $\mathrm{z} . \mathrm{z}$ can be much later than the beam waist $\mathrm{z}_{1}$.

As an example fig. 7 shows the emittance as a function of distance for the optimized simulation of Relativistic Heavy Ion Collider electron cooling injector. We see that at linac entrance the emittance has a drop which is the chromaticity effect and the beam waist is in front of linac entrance.

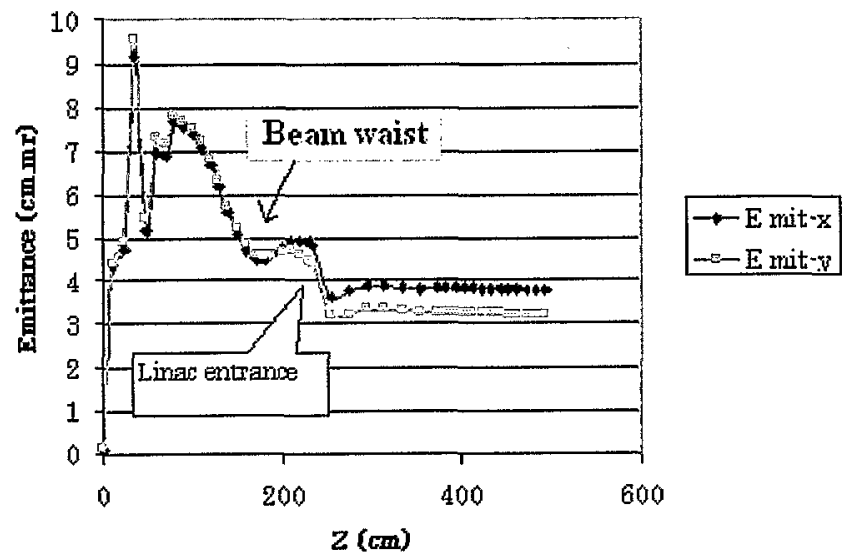

FIGURE 7. Emittance as a function of distance for optimized solenoid strength.

\section{CONCLUSIONS}

When the RF field in gun is low the cell lengths needs be accordingly short to have maximum accelerating efficiency. To the lowest order approximation this modification introduces beam divergence which is a linear function of beam position. When the emittance compensation system is used for this gun we must take into account the chromatic effect which may dominate the emittance growth. To well compensate the RF effect and chromatic effect equation (17) must be satisfied.

\section{REFERENCES}

1. B. E. Carlsten, New Photoelectric Injector Design for The Los Alamos National Laboratory XUV FEL Accelerator, NIM in Physics Research, A285 (1989), P313-319

2. O. D. Kellogg. Foundations of Potential Theory, P194, 1967 by Springer Verlag. Berlin, New York.

3. Luca Serafini, Envelope Analysis of Intense Relativistic Quasi-laminar Beams in RF Photoinjectors: A Theory of Emittance Compensation, Physical Review E, Vol. 55, Number 6, P7565 7590. (1997).

4. Kwang-Je Kim, RF and Space-Charge Effects in Laser-Driven RF Electron Guns, Nuclear Instruments and Methods in Physics Research A275 (1989) 201-218 


\title{
DISCLAIMER
}

This report was prepared as an account of work sponsored by an agency of the United States Government. Neither the United States Government nor any agency thereof, nor any of their employees, nor any of their contractors, subcontractors or their employees, makes any warranty, express or implied, or assumes any legal liability or responsibility for the accuracy, completeness, or any third party's use or the results of such use of any information, apparatus, product, or process disclosed, or represents that its use would not infringe privately owned rights. Reference herein to any specific commercial product, process, or service by trade name, trademark, manufacturer, or otherwise, does not necessarily constitute or imply its endorsement, recommendation, or favoring by the United States Government or any agency thereof or its contractors or subcontractors. The views and opinions of authors expressed herein do not necessarily state or reflect those of the United States Government or any agency thereof.

\section{FOR UNCLASSIFIED, UNLIMITED STI PRODUCTS}

Available electronically at-

\section{OSTI:}

http://www.osti.gov/bridge

Available for a processing fee to U.S. Department of Energy and its contractors, in paper from-

\author{
U.S. Department of Energy \\ Office of Scientific and Technical Information \\ P.O. Box 62 \\ Oak Ridge, TN 37831 \\ (865) 576-8401
}

Facsimile: (865) 576-5728

E-mail: reports@adonis.osti.gov

\section{National Technical Information Service (NTIS):}

Available for sale to the public from-

U.S. Department of Commerce

National Technical Information Service

5285 Port Royal Road

Springfield, VA 22131

(800) 553-6847

Facsimile: (703) 605-6900

Online ordering: http://www.ntis.gov/ordering.htm 